\title{
Mechanisms of optical angular momentum transfer to nematic liquid crystalline droplets
}

\author{
Tiffany A. Wood, Helen F. Gleeson, ${ }^{\text {a) }}$ Mark R. Dickinson, and Amanda J. Wright \\ Department of Physics and Astronomy, University of Manchester, Manchester, M13 9PL, United Kingdom
}

(Received 13 August 2003; accepted 1 April 2004; published online 7 May 2004)

\begin{abstract}
A detailed study is presented that evaluates the relative importance of wave plate behavior, scattering processes and absorption phenomena in transferring optical torque from circularly polarized light to optically trapped nematic droplets. A wide range of parameters is considered: droplet diameters between 1 and $15 \mu \mathrm{m}$, birefringence values from 0.15 to 0.26 and trapping beam powers from $50 \mathrm{~mW}$ to $400 \mathrm{~mW}$. Wave plate behavior is verified through the dependence of torque on droplet diameter and material birefringence. The dependence of the magnitude of the torque on material birefringence confirms the additional importance of the scattering mechanism. Absorption processes are found to be negligible. (C) 2004 American Institute of Physics.
\end{abstract}

[DOI: $10.1063 / 1.1753067]$

The transfer of spin angular momentum from light to a wide range of materials has been a subject of interest since the original experiments of Beth ${ }^{1}$ on quartz plates. The advent of optical trapping enabled Friese et al. to extend experiments to micron-sized calcite fragments that acted as wave plates. $^{2}$ Very recently, the optical manipulation of nematic liquid crystal droplets has been demonstrated, for example a fast optical switch was created by Juodkazis et al. by viewing an optically trapped droplet, rotating in circularly polarized light, between crossed polarizers. ${ }^{3}$ The same group also demonstrated that optical torque is transferred to a droplet through wave plate behavior, ${ }^{4}$ whereas Savchenko et al. proposed that angular momentum can be transferred to a liquid crystalline material through a light scattering process brought about by its birefringent properties. ${ }^{5}$ There is significant interest in using liquid crystalline materials in the transfer of optical torque, but there is some confusion surrounding the mechanisms responsible. This paper presents a detailed study of the relative importance of the possible mechanisms by which optical angular momentum might be transferred to a liquid crystalline droplet; wave plate behavior, scattering, absorption, and the optical Fréedericksz transition. We do not consider lensing effects as these will influence the trapping power rather than the transfer of angular momentum.

The optical torque transferred through wave plate behavior and scattering processes has been considered by Friese et $a .^{2}$ and Savchenko et al., ${ }^{5}$ respectively. The optical torque $\tau$ on a wave plate in circularly polarized light is modulated such that,

$$
\tau \propto E_{0}^{2}(1-\cos \Delta)
$$

where $E_{0}$ is the amplitude of the electric field component of the incident light. The retardance, $\Delta$, describes the phase shift between light field components experiencing the ordinary, $n_{0}$, and extraordinary, $n_{e}$, refractive indices of the birefringent medium-and depends on the thickness $t$ of the birefringent material and the wavelength $\lambda$,

\footnotetext{
a) Author to whom correspondence should be addressed; electronic mail: helen.gleeson@man.ac.uk
}

$$
\Delta=\frac{2 \pi}{\lambda} t\left(n_{0}-n_{e}\right) .
$$

The transfer of angular momentum to a liquid crystalline material through the light scattering process can be considered as follows. ${ }^{5}$ The separation of the extraordinary and ordinary paths in a birefringent medium acts to deviate the Poynting vector $\mathbf{S}$ of the light from the wave-vector $\mathbf{k}$ by an angle $\beta$. The torque $T_{S}$ applied to a nematic slab of volume $V$, refractive index $n$, by light of speed $c$ and intensity $I$, is

$$
T_{S}=V \beta \operatorname{In} / c \text {. }
$$

Spin angular momentum can also be transferred through photon absorption. ${ }^{5}$ Assuming that circularly polarized light of frequency $\omega$ is normally incident on a plane surface, the torque $T_{A}$ applied to a material with absorption coefficient $\alpha$, is,

$$
T_{A}=V \alpha I / \omega
$$

Finally, Santamato et al. observed the transfer of spin angular momentum to a homeotropically aligned nematic film $^{6}$ by a process known as self-induced stimulated light scattering (SISLS) and theoretically evaluated the rotation rate. ${ }^{7}$ It was shown that at intensities around $1 \mathrm{MW} / \mathrm{cm}^{2}$, nematic molecules are reoriented by the light induced Fréedericksz transition. Precession of molecules within the film occurred and elliptically polarized light emerged from the film, the ellipticity of which changed with increasing light intensity. Theoretically it was confirmed that the angular rotation rate of the film was non-linear with laser intensity and in some cases multi-valued. As far as we are aware the influence of this effect on nematic droplets has not yet been reported although intensities of the order of $1 \mathrm{MW} / \mathrm{cm}^{2}$ are commonly available within an optical trap.

The extent to which each mechanism is responsible for the transfer of optical torque to liquid crystalline droplets has not previously been investigated. With the anticipation that nematic droplets may be employed as optical switches and as rotors, it is important to understand the parameters upon which the optical torque is dependent. Thus we have evaluated the optical torque on nematic droplets over a large range of droplet sizes, material birefringence and trapping power. 
TABLE I. A summary of the nomenclature, birefringence (measured at 598 $\mathrm{nm}$ ) and transition temperatures for the nematic materials used.

\begin{tabular}{lcccc}
\hline \hline Name & E7 & E49 & ZLI 2008-100 & MDA-00-1444 \\
\hline$\Delta \mathrm{n}$ & 0.225 & 0.251 & 0.151 & 0.177 \\
$T_{c}\left({ }^{\circ} \mathrm{C}\right)$ & 60.5 & 100 & 68 & 98 \\
\hline \hline
\end{tabular}

A continuous wave $1064 \mathrm{~nm} \mathrm{Nd}: \mathrm{YVO}_{4}$ circularly polarized laser beam was used to form the optical trap. In order to trap droplets the laser beam was brought to a focus within a sample using an oil-immersion, Zeiss plan neofluar objective, numerical aperture 1.3. Microscope objectives are known for their high power losses; for the objective and wavelength used a $59 \pm 2 \%$ loss was expected ${ }^{8}$ and accounted for when calculating trapping powers. Nematic material dispersed in de-ionized water formed suspensions of nematic droplets after vigorous shaking; $70 \%$ of droplets had diameters between $1 \mu \mathrm{m}$ and $10 \mu \mathrm{m}$. Within the droplets, the molecules tend to align along one direction within the center of the droplet, the droplet director, whereas at the edge they lie parallel to the nematic-water interface. ${ }^{9}$ This director arrangement is referred to as the bipolar or dipole configuration. Suspensions were contained between a center cavity microscope slide and a cover-slip in a gap approximately 350 $\mu \mathrm{m}$ wide. A trap depth of $20 \mu \mathrm{m}$ from the cover-slip was maintained in order to minimize the influence of the sample walls on the fluid flow of the medium near to the droplet. ${ }^{10}$ The sample was viewed using white light, between crossed polarizers and a pinhole reduced the illumination on the sample to a spot $15 \mu \mathrm{m}$ in diameter to prevent surrounding particles from affecting measurements.

Plane polarized light experiencing only $n_{0}$, or $n_{e}$, traverses a birefringent medium with unchanged polarization and so does not pass the 2nd polarizer. The period of revolution of a droplet was established by measuring the time between 4 minima on the transmitted light signal collected by an avalanche photodiode, protected by a filter cutting out $1064 \mathrm{~nm}$ radiation. IMAQ vision development software from LabVIEW $^{11}$ was used to measure the diameter of each trapped particle from the image collected by a camera.

A constant trapping power of approximately $80 \mathrm{~mW}$ was used throughout the studies of the change in optical torque with particle size and material birefringence. Four nematic materials were studied with birefringence ranging from 0.15 to 0.26 at room temperature, each well below their nematicisotropic transition temperature $T_{c}$. The name, birefringence value measured at $586.9 \mathrm{~nm}$, and transition temperature of each material, taken from the manufacturer's ${ }^{12}$ data sheets, are shown in Table I. To investigate the optical torque dependence on the laser power, a $7.5 \mu \mathrm{m}$ diameter droplet with a material birefringence of 0.23 was trapped at powers ranging between $50 \mathrm{~mW}$ and $400 \mathrm{~mW}$.

The measured rotation rates of droplets with diameters in the range $1-10 \mu \mathrm{m}$ are presented on a logarithmic scale in Fig. 1(a) for the sample with birefringence of 0.23. Similar data were recorded for the other materials.

The torque $\Gamma_{\nu}$, due to drag on a solid sphere of diameter $d$, rotating within a medium of viscosity $\eta$ about its central axis with an angular frequency $\nu$, is given by ${ }^{13}$ axis with an angular frequency $\nu$, is given by ${ }^{13}$ monds, as a function of material birefringence.
Downloaded 04 May 2007 to 130.159 .254 .2 . Redistribution subject to AIP license or copyright, see http://apl.aip.

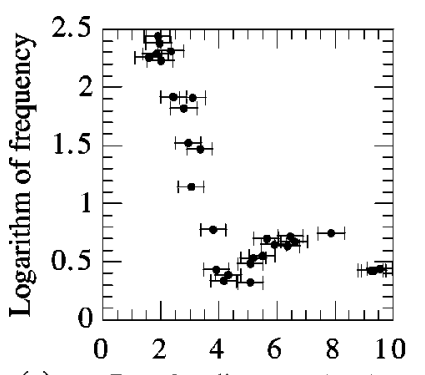

(a) Droplet diameter $(\mu \mathrm{m})$

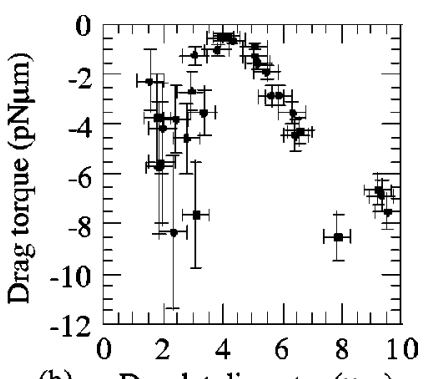

(b) Droplet diameter $(\mu \mathrm{m})$

FIG. 1. (a) The droplet angular rotation frequency as a function of the logarithm of the droplet diameter. (b) The viscous drag torque exerted on the droplet by the fluid medium, calculated from (a) for each particle diameter.

$$
\Gamma_{\nu}=-\pi d^{3} \eta \nu
$$

The drag on a fluid sphere may not follow Eq. (5) if appreciable circulation within the droplet occurs. ${ }^{14}$ However, since the viscosity of the nematic fluid is much greater than the surrounding water, Eq. (5) is a very good approximation of the drag torque on a nematic droplet.

With constant torque applied to a droplet, a linear relationship between the logarithm of the frequency and the particle diameter would be expected; however as is clear from Fig. 1(a) this is not the case. The viscous drag torque of the fluid on the particle calculated using Eq. (5) is presented in Fig. 1(b). The data modulate as expected for a viscous torque that counteracts optical torque originating from wave platelike behavior. Rotation maxima of the drag torque are observed at particle diameters around 2.5 and $8 \mu \mathrm{m}$. At these diameters a $\pi$ phase difference between the extraordinary and ordinary rays passing through the nematic droplet is probable. The dipole director orientation within a droplet lowers the effective birefringence of a droplet from the bulk birefringence value and thus modifies the modulation of torque associated with a uniaxial medium [Eq. (1)]. The effective birefringence is related only to the bulk birefringence and droplet size.

The average separation of the peaks of the modulating viscous drag torque was calculated for each nematic material and is plotted in Fig. 2. The peak separations change by approximately $2.5 \mu \mathrm{m}$ over the birefringence range 0.15 to 0.26 . The required increase in particle diameter for the retardance to remain constant appears in Fig. 2 to be inversely proportional to the material birefringence, in agreement with Eq. (2) and again confirming the wave-plate mechanism.

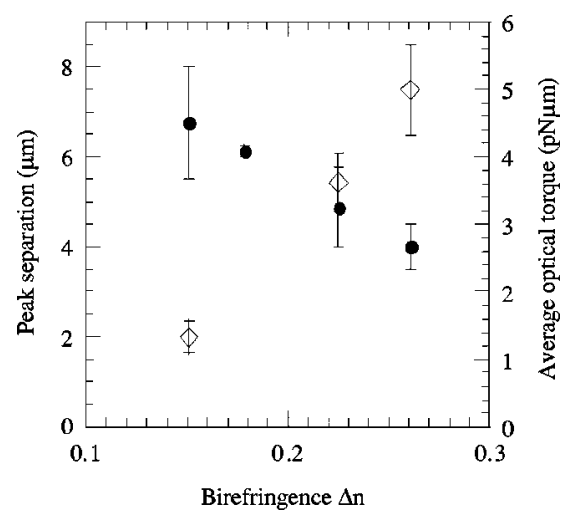

FIG. 2. The average particle size separation between peaks, filled circles, and the average magnitude of the torque (over all particle sizes), open dia- 


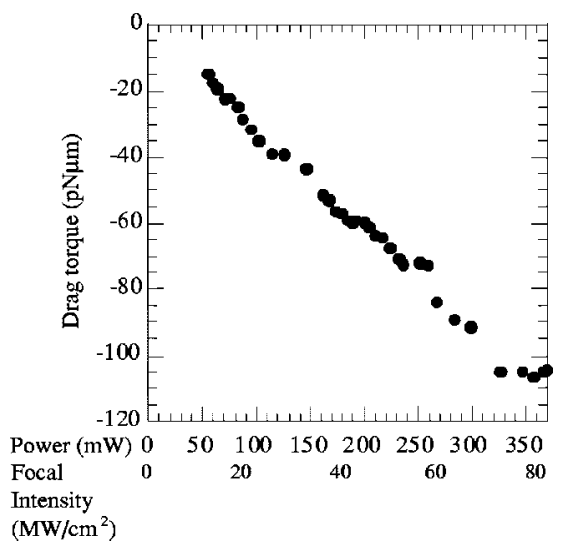

FIG. 3. Viscous drag torque for a $7.5 \mu \mathrm{m}$ diameter droplet as a function of the calculated power and intensity at the trap focus.

The average viscous drag torque calculated over the entire particle size range for each material is also shown in Fig. 2. There is a strong dependence on the material birefringence; the magnitude of the average viscous drag torque on droplets of material birefringence 0.26 is approximately five times greater than that on droplets of material birefringence 0.15 (both data sets are for equal beam powers). This effect, due to the increasing refractive index, allows more momentum to be transferred to the droplet. The scattering mechanism, with $\beta$ dependent on the birefringence, is responsible for the offset of $\sim 0.5 \mathrm{pN} \mu \mathrm{m}$ in Fig. 1(b) and is also shown to be a mechanism for angular momentum transfer to nematic droplets.

A linear dependence between the optical torque and the trapping beam power, for fixed birefringence and droplet diameter, is seen in Fig. 3. According to Eqs. (1), (3), and (4), the transfer of optical torque through wave plate behavior, scattering and absorption is in all cases proportional to the intensity of light incident on the droplet. We consider the last of these possible mechanisms as follows. From Eq. (4), the torque due to photon absorption on a $7.5 \mu \mathrm{m}$ diameter nematic droplet with absorption coefficient, $\alpha=10^{-4} \mathrm{~m}^{-1}$ (established using a spectrophotometer), for light of wavelength $1064 \mathrm{~nm}$ and intensity $I=10 \mathrm{MW} / \mathrm{cm}^{2}$, is around $10^{-6} \mathrm{pN} \mu \mathrm{m}$. Since a $10 \mathrm{pN} \mu \mathrm{m}$ torque on a droplet was measured through the drag force technique, absorption has a negligible contribution to the transfer of torque to a liquid crystal droplet. The linear dependence of the drag torque on the beam power (Fig. 3) confirms that any heating effects within the sample do not affect the angular momentum transferred to the droplet.

Additionally it can be seen from Fig. 3 that no non-linear effect, characteristic of SISLS, was observed with an increase in beam power. However, it was observed during experiments that the orientation of some droplets changed when entering the trapping beam. Droplets that were initially aligned with the droplet director out of plane, so that a dipole end was visible, reoriented as they entered the trapping beam until the droplet director was normal to the beam axis. The torque that realigns the droplet director from a 'vertical' to a 'horizontal' (in-plane) state could not be measured using the technique reported. It is possible that such torque could be evaluated by analysis of the outgoing polarization but we expect such measurements would be complicated by the rate of droplet reorientation. With trapping beam powers in excess of $1 \mathrm{MW} / \mathrm{cm}^{2}$, it is believed that the optical Freedericksz transition causes this reorienting effect and maintains in-plane orientation within the trap.

In summary, experiments have shown that waveplatelike behavior of nematic droplets and light scattering are predominately responsible for the transfer of optical torque to the droplets from the spin angular momentum of an optical trapping beam. Waveplate-like behavior is dependent on the size and birefringence of the nematic droplet, and causes the observed modulation in the transferred optical torque. Further, the modulation occurs with an amplitude of torque that is highly dependent on the birefringence of the nematic material; evidence of light scattering. A strong linear relationship between the torque and trapping beam power confirms the importance of light scattering and of waveplate-like behavior, and simultaneously eliminates the presence of nonlinear SISLS. The available torque due to photon absorption has been shown to be negligible for nematic droplets. Finally, experimental observations indicate that in the high intensities of an optical trap, the optical Freedericksz transition can cause a reorientation of the director of a nematic droplet toward the plane of polarization of the incident light.

A detailed understanding of which mechanism is dominant in the transfer of optical angular momentum to nematic droplets is important if they are to be used as optical switches or rotors. Nematic droplets can also be rotated and optically switched using electrodes. Since optical and electrical torque can both be applied to nematic droplets, ${ }^{15}$ new optical devices may be fabricated from such versatile anisotropic microstructures. This work identifies which mechanism for optical angular momentum transfer dominates for specific conditions.

The authors gratefully acknowledge the financial support of the Engineering and Physical Sciences Research Council, U.K., and the Jersey Education Department, G.B.

${ }^{1}$ R. Beth, Phys. Rev. 50, 115 (1936).

${ }^{2}$ M. E. K. Friese, T. A. Nieminen, N. R. Heckenberg, and H. RubinszteinDunlop, Nature (London) 394, 348 (1998).

${ }^{3}$ S. Juodkazis, M. Shikata, T. Takahashi, S. Matsuo, and H. Misawa, Jpn. J. Appl. Phys., Part 2 38, L518 (1999).

${ }^{4}$ S. Juodkazis, S. Matsuo, N. Murazawa, I. Hasegawa, and H. Misawa, Appl. Phys. Lett. 82, 4657 (2003).

${ }^{5}$ A. Yu. Savchenko, N. V. Tabiryan, and B. Ya. Zel'dovich, Phys. Rev. E 56, 4773 (1997)

${ }^{6}$ E. Santamato, B. Daino, M. Romagnoli, M. Settembre, and Y. R. Shen, Phys. Rev. Lett. 57, 2423 (1986).

${ }^{7}$ E. Santamato, M. Romagnoli, M. Settembre, and Y. R. Shen, Phys. Rev. Lett. 61, 113 (1988).

${ }^{8}$ K. Svoboda and S. M. Block, Rev. Biomol. Struct. 23, 247 (1994).

${ }^{9}$ S. Elston and R. Sambles, The Optics of Thermotropic Liquid Crystals (Taylor \& Francis, London, 1998), Vol. 1, p. 243.

${ }^{10}$ A. J. Wright, T. A. Wood, M. R. Dickinson, H. F. Gleeson, and T. Mullin, J. Mod. Opt. 50, 1521 (2003)

${ }^{11}$ National Instruments Corporation, 11500 North Mopac Expressway, Austin, Texas, USA.

${ }^{12}$ Merck KgaA, D-64271, Darmstadt, Germany.

${ }^{13}$ J. Happel and H. Brenner, Low Reynolds Number Hydrodynamics, 2nd ed. (Martinus Nijhoff Publishers, The Hague, 1983), p. 173.

${ }^{14} \mathrm{~J}$. Happel and H. Brenner, Low Reynolds Number Hydrodynamics, 2nd edition (Matrimus Nijhofff Publishers, The Hague, 1983), Vol. 1 p. 127.

${ }^{15}$ D. R. Cairns, M. Sibulkin, and G. P. Crawford, Appl. Phys. Lett. 78, 2643 (2001) 